Reprod. Nutr. Dévelop., 1988, 28 Suppl. n¹, 123-124

\title{
Effet d'une restriction alimentaire sur le flux de matière organique et d'azote dans le tube digestif des caprins
}

\author{
J. BRUN-BELLUT, M. HALBOUCHE, B. VIGNON
}

Laboratoire de Zootechnie, E.N.S.A.I.A.,

2, av. de la Forêt de Haye, 54500 Vandouvre, France.

Summary. A restriction in intake ( $75 \%$ of ad libitum) did not modify significantly the disappearance of energy in the rumen, the small and the large intestine. It decreased the $\mathrm{N}$ disappearance in the small intestine. Microbial synthesis was between 48 and $62 \mathrm{~g} \mathrm{~N} / \mathrm{kg}$ of organic matter apparently degraded in the rumen.

La diminution du niveau d'ingestion entraîne une amélioration de l'utilisation des aliments dans le tube digestif (Grovum et Williams, 1977). Nous avons étudié l'effet d'une restriction alimentaire modérée sur l'utilisation digestive des matières organique et azotées de la ration.

Matériel et méthodes. Trois chèvres adultes taries, équipées de fistules du rumen, du duodénum et de l'iléon, reçoivent une ration composée de foin de prairie permanente, de luzerne déshydratée et de pulpes de betteraves déshydratées (1/1/1 en matière sèche contenant $13 \%$ MAT). La ration est distribuée ad libitum pendant la période 1 , et en quantité limitée à $75 \%$ de la consommation mesurée précédemment pendant la période 2.

Les rations sont distribuées en deux repas: à 8 et à $16 \mathrm{~h} .3 \mathrm{~g}$ de papier imprégné d'oxyde de chrome et $50 \mathrm{ml}$ d'une solution à $30 \%$ de PEG sont administrés dans le rumen simultanément à la distribution des repas. Chaque période comprend 21 jours d'adaptation, 5 de mesure de digestibilités, 3 de prélèvements de contenus, effectués toutes les 2 heures pendant 24 heures à j27 pour l'iléon, j28 pour le duodénum et j29 pour le rumen.

Les teneurs en matière organique (MO) et azote total (NT) sont déterminées sur un échantillon moyen de la ration, des refus et des fécès et sur un échantillon moyen journalier des flux du duodénum et de l'iléon. Sur ces contenus les teneurs en ammoniac $\left(\mathrm{NH}_{3}\right)$, en PEG et en chrome sont mesurées. Les teneurs en azote bactérien du contenu de duodénum sont estimées à partir des taux de DAP, l'échantillon bactérien de référence étant isolé du contenu de rumen (Czerkawski, 1974). Les flux duodénaux et iléaux sont estimés par la méthode de Faichney (1980) sans correction par les taux de recouvrement fécaux. Les résultats sont comparés par analyse de variance, des lettres suivent des résultats différents $(P<0,05)$.

Résultats et discussion. Le taux de disparition de MO dans le rumen, l'intestin grêle, le gros intestin et dans l'ensemble du tube digestif n'est pas significativement modifié par la diminution des quantités ingérées (tabl. 1) même 
si la digestibilité apparente de MO dans le rumen a tendance à être plus élevée $(+8 \%)$. Les modifications de la digestibilité sont observées par Grovum et Williams (1977) pour des restrictions alimentaires supérieures à $30 \%$.

TABL. 1. - Quantités et taux de disparition de la matière sèche et de l'azote à différents niveaux du tube digestif.

\begin{tabular}{lcccc}
\hline & \multicolumn{2}{c}{ Matière organique } & \multicolumn{2}{c}{ Azote } \\
\cline { 2 - 5 } & ad libitum & restreint & ad libitum & restreint \\
\hline Quantités g/j & $1692 \mathrm{a}$ & $1272 \mathrm{~b}$ & $35,9 \mathrm{a}$ & $26,4 \mathrm{~b}$ \\
$\quad$ Ingérée & $1028 \mathrm{a}$ & $729 \mathrm{~b}$ & $51,9 \mathrm{a}$ & $37,1 \mathrm{~b}$ \\
$\quad$ Duodénum & 708 & 500 & 14,4 & 11,9 \\
Iléon & 558 & 397 & 13,1 & 9,4 \\
$\quad$ Fèces & 39,4 & 42,7 & $-44,4$ & $-40,7$ \\
\hline Taux de disparition (\%) & 18,9 & 18,0 & ${ }^{*} 72,1 \mathrm{a}$ & ${ }^{*} 67,6 \mathrm{~b}$ \\
$\quad$ Rumen & 8,9 & 8,1 & $* 6,1$ & ${ }^{*} 10,4$ \\
$\quad$ Duodénum & 67,2 & 68,8 & ${ }^{*} 74,9$ & ${ }^{*} 75,6$ \\
Iléon & & & 63,5 & 64,4 \\
$\quad$ Total & & & & \\
& & &
\end{tabular}

* \% du flux d'azote non ammoniacal entrant dans le duodénum.

La quantité d'azote non ammoniacal entrant dans le duodénum est supérieure de $40 \%$ à la quantité ingérée. La différence ou quantité nette d'azote recyclée correspond à 14 et $12 \mathrm{gN} / \mathrm{kg}$ de MOD pour les lots ad libitum et restreints (24 et $20 \mathrm{gN} / \mathrm{kg}$ MODR). Ramené au flux de MO pénétrant dans le duodénum, le recyclage net $n^{\prime}$ est pas différent d'un régime à l'autre ( $15 \mathrm{gN} / \mathrm{kg}$ MO duodénum).

La disparition de l'azote dans l'intestin grêle est supérieure chez les animaux nourris ad libitum, alors que la tendance est inverse dans le gros intestin (différence NS). La digestibilité totale de l'azote n'est pas significativement différente d'un régime à l'autre.

Les flux d'azote bactérien duodénal, estimés par le DAP, sont de 36 et $30 \mathrm{gN} / \mathrm{kg}$ de MOD pour les lots ad libitum et restreints. Ramené à la MODR le flux duodénal d'azote bactérien est de 62 et $48 \mathrm{~g}$ par $\mathrm{kg}$. Ces valeurs paraissent élevées pour des rations contenant $13 \%$ de MAT. Elles sont supérieures à celles estimées sur moutons et présentées dans la bibliographie, mais elles sont semblables à celles obtenues sur vaches laitières par Rode et al. (1985). Le recyclage net d'azote dans le rumen peut expliquer partiellement la différence de flux bactérien.

Czerkawski J. W., 1974. J. Sci. Fd. Agric., 25, 45-55.

Faichney G. J., 1980. J. agric. Sci. Camb., 94, 313-318.

Grovum W. L., Williams V. J., 1977. Br. J. Nutr., 38, 425-435.

Rode L. M., Weakley D. C., Satter L. D., 1985. Can. J. anim. Sci., 65, 101-111. 